\title{
Research on Methods of Active Steering Control Based on Receding Horizon Control
}

\author{
Jiwei Feng ${ }^{1}$, Chunjiang Bao ${ }^{1, *}$, Jian Wu ${ }^{1,2, *}$, Shuo Cheng ${ }^{2}{ }^{(\mathbb{D}}$, Guangfei $\mathrm{Xu}^{1}{ }^{1}$ and Shifu Liu ${ }^{1}$ \\ 1 School of Mechanical and Automotive Engineering, Liaocheng University, Liaocheng 252000, China; \\ jweifcheer@gmail.com (J.F.); 1620230105@lcu.edu.cn (G.X.); shifuliu239@gmail.com (S.L.) \\ 2 State Key Laboratory of Automotive Safety and Energy, Tsinghua University, Beijing 100084, China; \\ chengs16@mails.tsinghua.edu.cn \\ * Correspondence: baochunjiang@lcu.edu.cn (C.B.); wujian@lcu.edu.cn (J.W.)
}

Received: 24 July 2018; Accepted: 22 August 2018; Published: 27 August 2018

\begin{abstract}
Active steering technology is a key technology for automatic driving vehicles to achieve route tracking and obstacle avoidance and risk avoidance, and its performance will affect the stability control of the vehicle. For solving the stability control issues of vehicles, which have uncertainty in model and robustness in system, this paper proposes an active steering control method based on the receding horizon control model. It calculates the optimal control law by this method by using the real-time vehicle state so that it can compensate for the uncertainty caused by model mismatch, interference, etc. The design of the controller is implemented by using the yaw rate deviation of the vehicle as the input of the receding horizon linear quadratic controller model and then inputting the calculated superposition angle into the vehicle model in real time. We built a Simulink control model to implement co-simulation with CarSim to verify the control effect of the controller. In addition, we built a steering hardware-in-the-loop platform based on the LabVIEW RT system. The experimental results show that the active steering system adopting a receding horizon control method had better system robustness and robust stability.
\end{abstract}

Keywords: vehicle dynamics; receding horizon control; vehicle stability control; hardware-in-loop

\section{Introduction}

As an important component of vehicle stability control, the active steering system can determine the conditions that will occur according to the driving state of the vehicle. This provides an additional steering angle that is independent of the driver. It actively corrects the steering angle of the wheel to meet the steering requirements of the vehicle at low speeds, high speeds, and critical conditions and improves the operating stability of the vehicle. It has some advantages, such as quick response, not affecting the longitudinal dynamics of the vehicle, and the ability to ensure the stability of the vehicle under extreme conditions, etc. At present, the active steering technology is a key technology for automatic driving vehicles to achieve route tracking and obstacle avoidance and risk avoidance, and its performance will affect the active safety and driving performance. Therefore, many scholars have implemented research on active steering technologies [1-9].

Currently, the active steering control based on the classical control theory can achieve, to a large degree, the stability control of vehicles. Yu et al. designed the active steering controller based on fuzzy proportion integration differentiation (PID), which can improve the lateral stability of the vehicle under various lateral interferences [10]. Wang Ning designed the self-adaptive fuzzy PI controller that takes the deviation between the actual and the ideal yaw rate and the deviation between the actual and the ideal sideslip angle as the input; this enhances the driving stability of the vehicle [11]. By fully considering the limitations, such as ground attachment, steering, and braking, etc., Li Jing et al. 
designed the chassis integrated controller by adopting fuzzy PID. This has a good effect on the stable control of the vehicle [12]. However, PID control is applied mainly to model-free engineering control and its parameters are usually regulated by experience. Therefore, it is difficult to obtain optimal PID parameters and its robustness for all operating conditions is not good. These active steering controls based on the classical control theory are unable to inhibit the uncertainty of the steering system and the interference caused by nonlinear factors.

As for the problem that the control methods based on experiences are not able to effectively inhibit the noise and interference of the system, some scholars have further developed active steering control by adopting the modern control theory. Li et al. proposed a control strategy based on active disturbance rejection, which has a better control effect than PID and has a higher capability in route tracking and disturbance rejection [13]. Sang Nan et al. designed the active disturbance rejection controller for active steering of the front wheels, which improves the control stability of the vehicle [14]. Huo et al. implemented an optimization to the control algorithm and designed the optimized back-propagation (BP) network controller based on a genetic algorithm [15]. These controllers allow the vehicle to achieve good stability control.

To improve the robustness of the system, Wu Jian et al. designed the active steering general internal model robust controller for solving the contradiction between the performance and robustness of the controller [16]. Chen et al. implemented the design of active steering controller for assisting drivers in lane retention based on the control method, which improves the robust performance of the system [17]. Zhao et al. studied the active steering system based on the hybrid control of $H_{2} / H_{\infty}$, which enhances the capacity of resisting disturbance of the system by using $H_{\infty}$, and improves the turning stability and maneuverability of the vehicle through optimization implemented by $\mathrm{H}_{2}$ [18]. Nam et al. designed the active steering robust controller that is applicable for electric vehicles. The controller effectively resists external disturbances to guarantee the robustness of the system [19]. Ji et al. designed the active steering robust controller on the basis of model uncertainty and external disturbance [20]. By taking into consideration factors such as model uncertainties, various external disturbances, sensor noise, etc., $\mathrm{Xu} Z$ Zhijiang designed the yaw rate robust controller of systems based on $\mu$ comprehensive robust control, which can effectively improve the active safety and operating stability of vehicles [21]. Aiming at a two-degrees of freedom vehicle model of a linear vehicle model with yaw and sideways, Said Mammer et al. designed an active steering controller for the control, which improves the performance and robustness of the controller through making up the parameter uncertainty of the vehicle [22].

Good progress has been made for the above active steering control methods. However, there is a gap between the steering control model adopted by these researchers and the actual steering system and some have not been tested to verify the effectiveness of the algorithm. We designed an active steering controller based on a receding horizon control to solve the problems of external disturbance and model uncertainty. This method has been applied in many fields [23]. This method applies the first component of the optimal solution $U_{k}^{*}$ at the current moment $k$ to the system; at the next moment of $k+1$, it applies the new measured value $y(k+1)$ as the initial condition to re-predict the future output of the system and solve the optimization problem. Through this method, the external disturbance in the measured value or the error information of the model is effectively used to promote the performance stability of the system. This paper designed an active steering experiment platform based on LabVIEW RT on the basis of simulation and verification of the algorithm, implemented experimental verification, and simultaneously implemented a comparison with the control effect of the PID controller. The results show that the receding horizon controller had a faster response and better control effect than the PID controller. Especially in the process of actual use, it could improve the robustness of the system, had a good effect on the suppression of external noise, and could better guarantee the stability of the vehicle. 


\section{Control Strategy of Receding Horizon}

The overall control strategy of the receding horizon control is shown in Figure 1. It includes the vehicle's modules such as the two-degrees of freedom vehicle module, tire model, target value calculation module, controller, etc. The target value calculation module provided the target value of the variable of the system in real time, and the receding horizon controller directly provided the superposition steering angle of the steering of the front wheel. The final control steering angle was then obtained after the superimposition with the target steering angle functions together with the vehicle to achieve stability control of the vehicle.

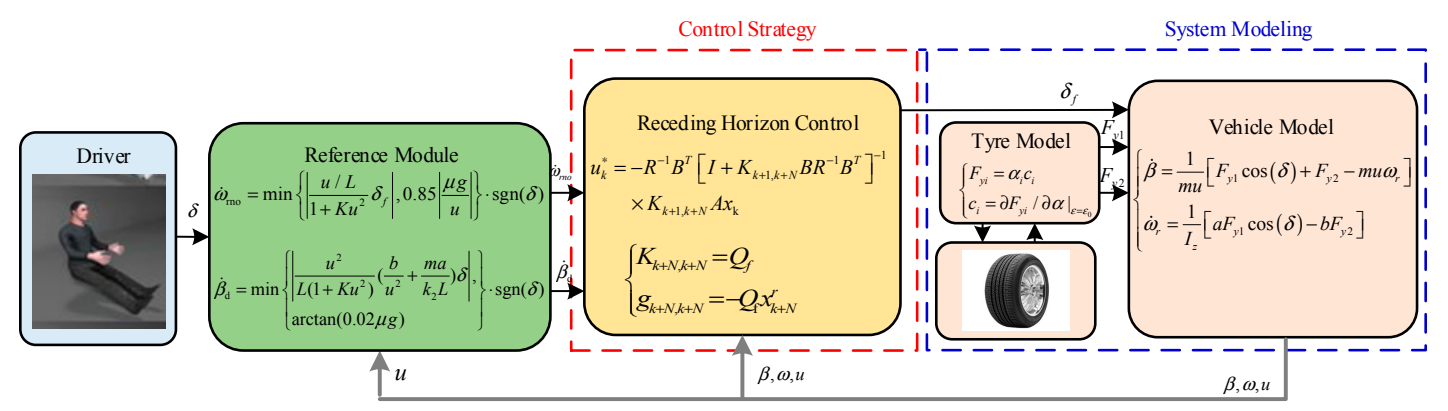

Figure 1. Global control strategy for receding horizon control.

\subsection{Vehicle Control Model}

In this paper, the stability control of vehicle active steering was studied. A simplified model of a vehicle with two degrees of freedom is presented in Figure 2 [24].

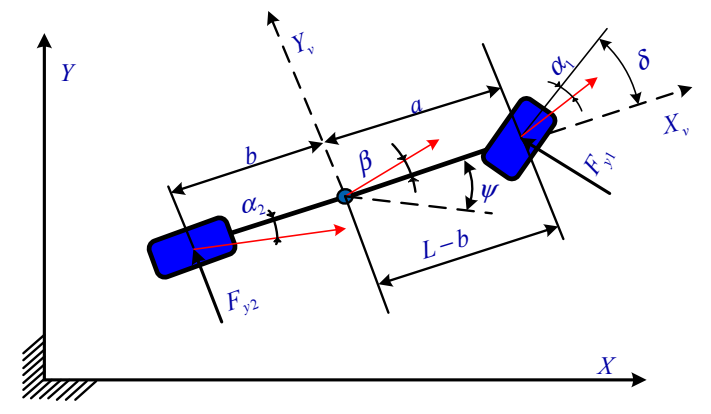

Figure 2. Two-degrees of freedom vehicle model.

The kinetic equation of the vehicle is:

$$
\left\{\begin{array}{l}
\dot{\beta}=\frac{1}{m v_{x}}\left[F_{y 1} \cos (\delta)+F_{y 2}-m v_{x} \omega_{r}\right] \\
\dot{\omega}_{r}=\frac{1}{I_{z}}\left[a F_{y 1} \cos (\delta)-b F_{y 2}\right]
\end{array}\right.
$$

Among them, $m$ is the mass of the whole vehicle; $v_{x}$ is the longitudinal velocity of the vehicle; $\beta$ is the sideslip angle of the vehicle; $\omega_{r}$ is the yaw rate of the vehicle; $\delta$ is the front-wheel steering angle of the vehicle; $a$ is the distance from the front wheel of the vehicle to the mass center; $b$ is the distance from the rear wheel of the vehicle to the mass center; $I_{z}$ is the moment of inertia of the vehicle rounding the z-axis; $F_{y 1}$ is the tire lateral force of the front wheel of the vehicle; $F_{y 2}$ is the tire lateral force of the rear wheel of the vehicle.

The magic tire model can describe the non-linear characteristics of a tire. The formula for magic tires is:

$$
Y(x)=D \sin \{C \arctan [B x-E(B \alpha-\arctan B x)]\}
$$


where $D$ is the peak factor, $C$ is the shape factor, $B$ is the stiffness factor, and $E$ is the curvature factor. The lateral force of the tire in the formula is:

$$
F_{y}=D_{y} \sin \left\{C_{y} \arctan \left[B_{y} \alpha-E_{y}\left(B_{y} \alpha-\arctan \left(B_{y} \alpha\right)\right)\right]\right\}
$$

where

$$
\begin{aligned}
& C_{y}=a_{0} ; D=a_{1} F_{z}^{2}+a_{2} F_{z} \\
& B_{y}=\frac{a_{3} \sin \left[2 \arctan \left(F_{z} / a_{4}\right)\right]}{C_{y} D_{y}} \\
& E_{y}=a_{5} F_{z}+a_{6}
\end{aligned}
$$

The data measured by experiment in the formula is shown in Table 1 [25].

Table 1. Tire Magic Formula model parameters.

\begin{tabular}{ccccccc}
\hline$a_{0}$ & $a_{1}$ & $a_{2}$ & $a_{3}$ & $a_{4}$ & $a_{5}$ & $a_{6}$ \\
\hline 1.75 & 0 & 1000 & 1289 & 7.11 & 0.0053 & 0.1925 \\
\hline
\end{tabular}

The wheel side deflection angle of the vehicle is obtained by measuring the parameters and estimating:

$$
\left\{\begin{array}{l}
\alpha_{1}=\left(\beta+\frac{a \omega_{r}}{v_{x}}-\delta\right) \cos \delta \\
\alpha_{2}=\beta-\frac{b \omega_{r}}{v_{x}}
\end{array}\right.
$$

where $\alpha_{1}$ is the front wheel side deflection angle and $\alpha_{2}$ is the rear wheel side bias force.

It is difficult to solve the system dynamics equation by using the nonlinear magic tire formula. Therefore, the local linearization method is used to transform the nonlinear problem into a linear problem. The real-time status of the vehicle is $\varepsilon_{0}$ and the tire side deflection angle of the vehicle is $\alpha_{i}$. The lateral force of the tire can be written as follows:

$$
\left\{\begin{array}{l}
F_{y i}=\alpha_{i} c_{i} \\
c_{i}=\partial F_{y i} /\left.\partial \alpha\right|_{\varepsilon=\varepsilon_{0}}
\end{array}\right.
$$

Substituting Equation (5) into Equation (1), we have the following:

$$
\dot{x}(t)=A x(t)+B u(t)+B_{\omega} \omega(t)
$$

where $x$ is the system variables $x=\left[\beta, \omega_{r}\right]^{T} ; A$ is the state matrix; $u$ is the vehicle's front wheel stacking corner; $B$ is the control matrix; $\omega_{t}$ is the interference input; $B_{\omega}$ is the corresponding interference input matrix. Road signal input can be written as $\omega=s_{0} \delta(t)$ where $s_{0}$ represents the disturbance intensity coefficient and $\delta(t)$ is the pavement disturbance noise.

The coefficient matrix $A, B, B_{\omega}$ can be written and $G_{0}$ is the road roughness factor.

$$
\begin{gathered}
A=\left[\begin{array}{cc}
\frac{1}{m v_{x}}\left(c_{1}+c_{2}\right) & \frac{1}{m v_{x}}\left(a c_{1}-b c_{2}\right)-v_{x} \\
\frac{a c_{1}-b c_{2}}{I_{z} v_{x}} & \frac{a^{2} c_{1}+b^{2} c_{2}}{I_{z} v_{x}}
\end{array}\right] \\
B=\left[\begin{array}{c}
\frac{-c_{1}}{m} \\
\frac{-a c_{1}}{I_{z}}
\end{array}\right] ; B_{\omega}=\left[\begin{array}{c}
0 \\
2 \pi \sqrt{G_{0} u}
\end{array}\right]
\end{gathered}
$$

\subsection{The Target Quantity}

The linear two-degrees-of-freedom vehicle model is used as the ideal reference model. In the case of angular input, the two-DOF model can produce a steady state response [26]. The desired yaw rate is 


$$
\omega_{\text {rno }}=\frac{v_{x} / L}{1+K v_{x}^{2}} \delta
$$

The expectation of the centroid side deflection can be written as follows:

$$
\beta_{r n o}=\frac{v_{x}^{2}}{L\left(1+K v_{x}^{2}\right)}\left(\frac{b}{v_{x}^{2}}+\frac{m a}{k_{2} L}\right) \delta
$$

The upper-type $L$ represents the front and rear wheelbase of the vehicle and $k_{1}, k_{2}$ are the lateral stiffness of the front and rear wheels of the vehicle, respectively.

The stability factor is as follows:

$$
K=\frac{m}{L^{2}}\left(\frac{a}{k_{2}}-\frac{b}{k_{1}}\right)
$$

The above yaw angle velocity and the expectation of centroid side deflection do not consider some uncertain factors, such as tire, road surface attachment, etc. These will lead to control deviation, especially when the vehicle is in the critical state of attachment limit. Revising the expected value is necessary. The desired yaw rate correction can be written as follows:

$$
\dot{\omega}_{r n o}=\min \left\{\left|\frac{v_{x} / L}{1+K v_{x}^{2}} \delta_{f}\right|,\left|\frac{\mu g}{v_{x}}\right|\right\} \cdot \operatorname{sgn}(\delta)
$$

The motor vehicle may have entered the non-linear zone when the upper limit is not reached. Therefore, the upper-type correction can be described as follows:

$$
\dot{\omega}_{r n o}=\min \left\{\left|\frac{v_{x} / L}{1+K v_{x}^{2}} \delta_{f}\right|, 0.85\left|\frac{\mu g}{v_{x}}\right|\right\} \cdot \operatorname{sgn}(\delta)
$$

The correction of the lateral deflection angle of the desired centroid can be written as follows:

$$
\dot{\beta}_{d}=\min \left\{\left|\frac{v_{x}^{2}}{L\left(1+K v_{x}^{2}\right)}\left(\frac{b}{v_{x}^{2}}+\frac{m a}{k_{2} L}\right) \delta\right|, \arctan (0.02 \mu g)\right\} \cdot \operatorname{sgn}(\delta)
$$

\subsection{Receding Horizon Control Scheme}

The receding horizon control predicts the system state in the future finite period according to the current sampling time and solves the optimal control quantity where $k$ is the current moment and $j$ is the time interval. The prediction time domain is expressed by $N$, the control time domain is represented by $m$, and $i_{f}=k+N$ represents the terminal moment of receding horizon prediction.

The discrete equation can be obtained by discretization of the Formula (6):

$$
\left\{\begin{array}{l}
\Delta x(k+1)=A_{d} \Delta x(k)+B_{d} \Delta u(k)+B_{\omega d} \Delta \omega(k) \\
z(k)=C_{d} \Delta x(k)+z(k-1)
\end{array}\right.
$$

where

$$
\left\{\begin{array}{l}
\Delta x(k)=x(k)-x(k-1) \\
\Delta u(k)=u(k)-u(k-1) \\
\Delta \omega(k)=\omega(k)-\omega(k-1) \\
C_{d}=\left[\begin{array}{ll}
1 & 0 \\
0 & 1
\end{array}\right]
\end{array}\right.
$$

The coefficient matrix $A_{d}, B_{d}, B_{\omega d}$ can be written as follows: 


$$
\left\{\begin{array}{l}
A_{d}=e^{A T_{s}} \\
B_{d}=\int_{0}^{T_{s}} e^{A t} d t \cdot B \\
B_{\omega d}=\int_{0}^{T_{s}} e^{A t} d t \cdot B_{\omega}
\end{array}\right.
$$

$T_{S}$ and $e^{A_{c} t}$ are expressed as the controller cycle and the state transition matrix of a continuous system. The receding horizon control solution makes the following assumptions:

The amount of system control outside the control time domain remains unchanged, namely:

$$
a=1, \Delta u(k+i)=0, i=m, m+1, \cdots, N-1
$$

The detectable interference remains unchanged after the current control moment; namely

$$
a=1, \Delta \omega_{1}(k+i)=\Delta \omega_{2}(k+i)=0, i=1,2, \cdots, N-1
$$

The discrete time-invariant system of a referenced predictive form:

$$
\begin{gathered}
x_{k+j+1 \mid k}=A_{d} x_{k+j \mid k}+B_{d} u_{k+j \mid k}+B_{\omega d} \omega_{k+j \mid k} \\
z_{k+j \mid k}=C_{d} x_{k+j \mid k}
\end{gathered}
$$

Performance metrics can be written as follows:

$$
J\left(x_{k \mid k}, x^{r}, u_{k+\times \mid k}\right)=\sum_{j=0}^{i_{f}-k-1}\left[\left(x_{k+j \mid k}-x_{k+j \mid k}^{r}\right)^{T} Q\left(x_{k+j \mid k}-x_{k+j \mid k}^{r}\right)+u_{k+j \mid k}^{T} R u_{k+j \mid k}\right]+\left(x_{i_{f} \mid k}-x_{i_{f} \mid k}^{r}\right)^{T} Q_{f}\left(x_{i_{f} \mid k}-x_{i_{f} \mid k}^{r}\right)
$$

where

$$
\left\{\begin{array}{l}
Q=C_{d}^{T} \bar{Q} C_{d} \\
Q_{f}=C_{d}^{T} \overline{Q_{f}} C_{d}
\end{array}\right.
$$

The Hamilton function can be written as follows:

$$
H_{k+j \mid k}=\left[\left(x_{k+j \mid k}-x_{k+j \mid k}^{r}\right)^{T} Q\left(x_{k+j \mid k}-x_{k+j \mid k}^{r}\right)+u_{k+j \mid k}^{T} R u_{k+j \mid k}\right]+p_{k+j+1 \mid k}^{T}\left[A_{d} x_{k+j \mid k}+B_{d} u_{k+j \mid k}\right]
$$

Through the Formula (19) deviation:

$$
\left\{\begin{array}{l}
p_{k+j \mid k}=\frac{\partial H_{k+j \mid k}}{\partial x_{k+j \mid k}}=2 Q\left(x_{k+j \mid k}-x_{k+j \mid k}^{r}\right)+A^{T} p_{k+j+1 \mid k} \\
p_{i_{f} \mid k}=\frac{\partial h\left(x_{i_{f} \mid k, i_{f}}\right)}{\partial x_{i_{f} \mid k}}=2 Q_{f}\left(x_{i_{f} \mid k}-x_{i_{f} \mid k}^{r}\right)
\end{array}\right.
$$

where

$$
h\left(x_{i_{f}}, i_{f}\right)=\left(x_{i_{f}}-x_{i_{f}}^{r}\right)^{T} Q_{f}\left(x_{i_{f}}-x_{i_{f}}^{r}\right)
$$

so that $\frac{\partial H_{k+j \mid k}}{\partial u_{k+j \mid k}}=0$ can get the following:

$$
\frac{\partial H_{k+j \mid k}}{\partial u_{k+j \mid k}}=2 R u_{k+j \mid k}+B^{T} p_{k+j+1 \mid k}
$$

The optimal control output can be written as:

$$
u_{k+j \mid k}^{*}=-\frac{1}{2} R^{-1} B^{T} p_{k+j+1 \mid k}
$$

where 


$$
p_{k+j+1 \mid k}=2 K_{k+j, i_{f} \mid k} x_{k+j \mid k}+2 g_{k+j, i_{f} \mid k}
$$

The boundary conditions are

$$
\left\{\begin{array}{l}
K_{i_{f}, i_{f} \mid k}=Q_{f} \\
g_{i_{f}, i_{f} \mid k}=-Q_{f} x_{i_{f} \mid k}^{r}
\end{array}\right.
$$

The Formulas (18) and (19) are substituted with (26), and Formula (25) is substituted for $u_{k+j}$ :

$$
p_{k+j+1 \mid k}=2 K_{k+j+1, i_{f} \mid k}\left(A_{d} x_{k+j+1 \mid k}-\frac{1}{2} B_{d} R^{-1} B^{T} p_{k+j+1 \mid k}\right)+2 g_{k+j+1, i_{f} \mid k}
$$

The solution for Formula (28) can be obtained as follows:

$$
p_{k+j+1 \mid k}=\left[I+K_{k+j+1, i_{f} \mid k} B_{d} R^{-1} B^{T}\right]^{-1}\left[2 K_{k+j+1, i_{f} \mid k} A_{d} x_{k+j+1 \mid k}+2 g_{k+j+1, i_{f} \mid k}\right]
$$

Formula (29) is used in (25) to obtain the optimal receding horizon solution for the next N-step:

$$
u_{k+j \mid k}^{*}=-R^{-1} B^{T}\left[I+K_{k+j+1, i_{f} \mid k} B R^{-1} B^{T}\right]^{-1} \times\left[K_{k+j+1, i_{f} \mid k} A x_{k+j \mid k}+g_{k+j+1, i_{f} \mid k}\right]
$$

Formula (29) is in the Formula (23):

$$
p_{k+j \mid k}=2 Q\left(x_{k+j}-x_{k+j}^{r}\right)+A^{T}\left[I+K_{k+j+1, i_{f} \mid k} B_{d} R^{-1} B^{T}\right]^{-1}\left[2 K_{k+j+1, i_{f} \mid k} A_{d} x_{k+j+1 \mid k}+2 g_{k+j+1, i_{f} \mid k}\right]
$$

where

$$
\left\{\begin{aligned}
K_{k+j, i_{f} \mid k} & =A^{T}\left[I+K_{k+j+1, i_{f} \mid k} B R^{-1} B^{T}\right]^{-1} K_{k+j+1, i_{f} \mid k} A+Q \\
& =A^{T} K_{k+j+1, i_{f} \mid k} A-A^{T} K_{k+j+1, i_{f} \mid k} B\left(R+B^{T} K_{k+j+1, i_{f} \mid k} B\right)^{-1} B^{T} K_{k+j+1, i_{f} \mid k} A+Q \\
g_{k+j, i_{f} \mid k} & =A^{T}\left[I+K_{k+j+1, i_{f} \mid k} B R^{-1} B^{T}\right]^{-1} g_{k+j+1, i_{f} \mid k}-Q x_{k+j \mid k}^{r}
\end{aligned}\right.
$$

The performance index of the receding horizon based on current time $k$ and predictive interval $[k, k+N]$ is as follows:

$$
J\left(x_{k \mid k}, x^{r}, u_{k+|| k}\right)=\sum_{j=0}^{N-1}\left[\left(x_{k+j \mid k}-x_{k+j \mid k}^{r}\right)^{T} Q\left(x_{k+j \mid k}-x_{k+j \mid k}^{r}\right)+u_{k+j \mid k}^{T} R u_{k+j \mid k}\right]+\left(x_{k+N \mid k}-x_{k+N \mid k}^{r}\right)^{T} Q\left(x_{k+N \mid k}-x_{k+N \mid k}^{r}\right)
$$

The optimal solution of the receding horizon control for the predictive interval $[k, k+N]$ is as follows:

$$
u_{k+j \mid k}^{*}=-R^{-1} B^{T}\left[I+K_{k+j+1, k+N \mid k} B R^{-1} B^{T}\right]^{-1} \times\left[K_{k+j+1, k+N \mid k} A x_{k+j \mid k}+g_{k+j+1, k+N \mid k}\right]
$$

where

$$
\begin{gathered}
\left\{\begin{array}{r}
K_{k+j, k+N \mid k=} A^{T}\left[I+K_{k+j+1, k+N \mid k} B R^{-1} B^{T}\right]^{-1} K_{k+j+1, k+N \mid k} A+Q \\
g_{k+j, k+N \mid k}=A^{T}\left[I+K_{k+j+1, k+N \mid k} B R^{-1} B^{T}\right]^{-1} g_{k+j+1, k+N \mid k}-Q x_{k+j \mid k}^{r}
\end{array}\right. \\
\left\{\begin{array}{l}
K_{k+N, k+N \mid k}=Q_{f} \\
g_{k+N, k+N \mid k}=-Q_{f} x_{k+N \mid k}^{r}
\end{array}\right.
\end{gathered}
$$

Based on the current time $k$, the optimal control amount of the receding horizon is $u_{k}$ and the optimal control quantity is expressed as $u_{k+i \mid k}, i=0,1, \cdots k+N-1$. At the same time, make $j=0$ :

$$
u_{k \mid k}^{*}=-R^{-1} B^{T}\left[I+K_{k+1, k+N \mid k} B R^{-1} B^{T}\right]^{-1} \times\left[K_{k+1, k+N \mid k} A x_{k+j \mid k}+g_{k+1, k+N \mid k}\right]
$$


$K_{k+1, k+N \mid k}$ and $g_{k+1, k+N \mid k}$ can be obtained by Formula (32).

The receding horizon controls an expression that simplifies the optimal control by deleting a reference value:

$$
u_{k+j}^{*}=-R^{-1} B^{T}\left[I+K_{k+j+1, i_{f}} B R^{-1} B^{T}\right]^{-1} \times\left[K_{k++j 1, i_{f}} A x_{k+j}+g_{k++j+1, i_{f}}\right]
$$

where

$$
\left\{\begin{array}{l}
K_{k+j, i_{f} \cdot}=A^{T}\left[I+K_{k+j+1, i_{f}} B R^{-1} B^{T}\right]^{-1} K_{k+j+1, i_{f}} A+Q \\
g_{k+j, i_{f}}=A^{T}\left[I+K_{k+j+1, i_{f}} B R^{-1} B^{T}\right]^{-1} g_{k+j+1, i_{f}}-Q x_{k+j}^{r}
\end{array}\right.
$$

Meanwhile, $K_{i_{f}, i_{f}}=Q_{f}, g_{i_{f}, i_{f}}=-Q_{f} x_{i_{f}}^{r}$. Replacing $u_{k \mid k}$ and $K_{k+1, k+N}$ with $u_{k}$ and $K_{k+1, k+N}$ can be:

$$
u_{k}^{*}=-R^{-1} B^{T}\left[I+K_{k+1, k+N} B R^{-1} B^{T}\right]^{-1} \times\left[K_{k+1, k+N} A x_{k}+g_{k+1, k+N}\right]
$$

where

$$
\left\{\begin{array}{l}
K_{k+j, k+N .}=A^{T}\left[I+K_{k+j+1, k+N} B R^{-1} B^{T}\right]^{-1} K_{k+j+1, k+N} A+Q \\
g_{k+j, k+N}=A^{T}\left[I+K_{k+j+1, k+N} B R^{-1} B^{T}\right]^{-1} g_{k+j+1, k+N}-Q x_{k+j}^{r}
\end{array}\right.
$$

Meanwhile,

$$
\left\{\begin{array}{l}
K_{k+N, k+N .}=Q_{f} \\
g_{k+N, k+N}=-Q_{f} x_{k+N}^{r}
\end{array}\right.
$$

The optimal control output is obtained by solving the above process:

$$
u_{k}^{*}=-R^{-1} B^{T}\left[I+K_{k+1, k+N} B R^{-1} B^{T}\right]^{-1} \times K_{k+1, k+N} A x_{k}
$$

\section{Simulation Analysis}

Figures 3 and 4 provide the state parameter curves of the vehicle under the three conditions of receding horizon control, PID control, and no control. Figure 3a shows the lateral displacement under the three conditions, from which we can see that the vehicle without control had a large delay in the route tracking when it passed through the double-lane-change route, it formed a steeper route with a larger magnitude, and the vehicle was in a state of instability. The vehicles provided with receding horizon control and PID control both completed the work condition of a double-lane-change route. However, the vehicle provided with receding horizon control had a tracking that was closer to the ideal tracking. During the emergency steering process, maximum lateral displacement deviation between the PID control and receding horizon control was nearly $1 \mathrm{~m}$. In the process of vehicle stability control, the vehicle provided with receding horizon control had completed emergency obstacle avoidance when driving to $350 \mathrm{~m}$. However, the PID-controlled vehicle did not complete emergency obstacle avoidance when it drove nearly $450 \mathrm{~m}$. Figure $3 \mathrm{~b}$ shows the comparison of the steering wheel angles from which we can see that the vehicle without control had a larger steering wheel angle input and the vehicle provided with receding horizon control had a smaller steering wheel manipulation than the vehicle provided with PID control.

From Figure 4a, we can see that the vehicle without control had a very large sideslip angle and the vehicle had lost stability; there was a reduction in the sideslip angle of the vehicle provided with receding horizon control compared with the vehicle provided with the PID control. In Figure $4 \mathrm{~b}$, it implements the comparison of yaw rate under the three control states. To verify that the proposed receding horizon control has anti-interference capability, noise was added to the simulation; it can be seen that there was a higher change in the yaw rate without control and after $4 \mathrm{~s}$ the vehicle lost stability. Although it can effectively track the yaw rate for the vehicle provided with PID control, 
the control effect of PID was inferior to that of receding horizon control under extreme conditions. The receding horizon control was able to resist the impact of model uncertainties and it could better track the ideal operating characteristics of the vehicle.
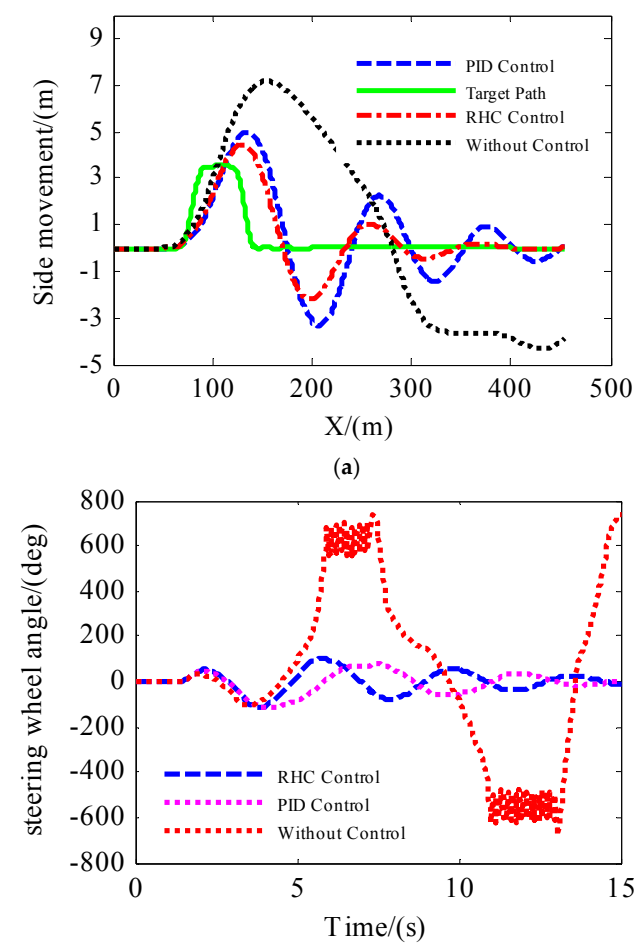

(b)

Figure 3. (a) Lateral displacement; (b) Steering wheel corner.

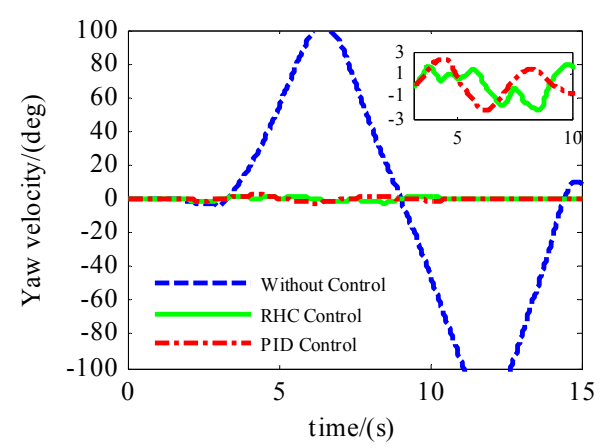

(a)

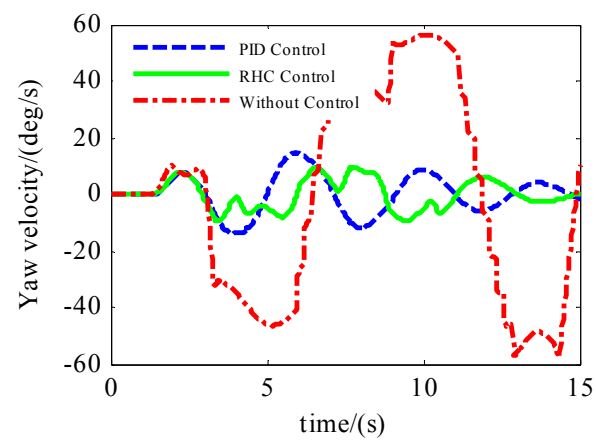

(b)

Figure 4. (a) Sideslip angle; (b) Yaw rate. 


\section{HIL Implementation}

In this section, the controller was embedded on the hardware-in-loop (HIL) platform to verify the true performance of the controller [27-29]. In the HIL, although the vehicle simulation model or commercial vehicle simulation software was used to replace the real vehicle, the actuator and control system were complete Therefore, it has been widely used in vehicle stability control and automatic driving control logic verification by vehicle factories and research institutions. In the HIL system established in this paper, CarSim embedded in the PXI real-time control system was used for vehicle simulation and to provide road information. Through PXI's CAN card, the vehicle state parameters, such as steering angle, yaw rate, speed, and so on, were transmitted to the active steering controller based on dSPACE. In ASPACE, vehicle state parameter information received from PXI was used to calculate the superimposed target active steering angle in real time according to the active steering controller designed in this paper. The target rotation angle was sent to the bottom steering controller to drive the steering motor to execute the target rotation angle. The Bosch angle sensor was used to collect the real steering angle and transmit the steering angle to CarSim. Through the above steps, a steering HIL was realized.

Under the conditions of simulating the original work condition, it analyzed and compared the stability of active steering under the strategy of receding horizon control and PID control. In this paper, the initial speed of the hardware-in-the-loop experiment was $120 \mathrm{~km} / \mathrm{h}$, and it adopted a double-lane-change route. The hardware-in-the-loop experiment platform for active steering is shown in Figure 5.

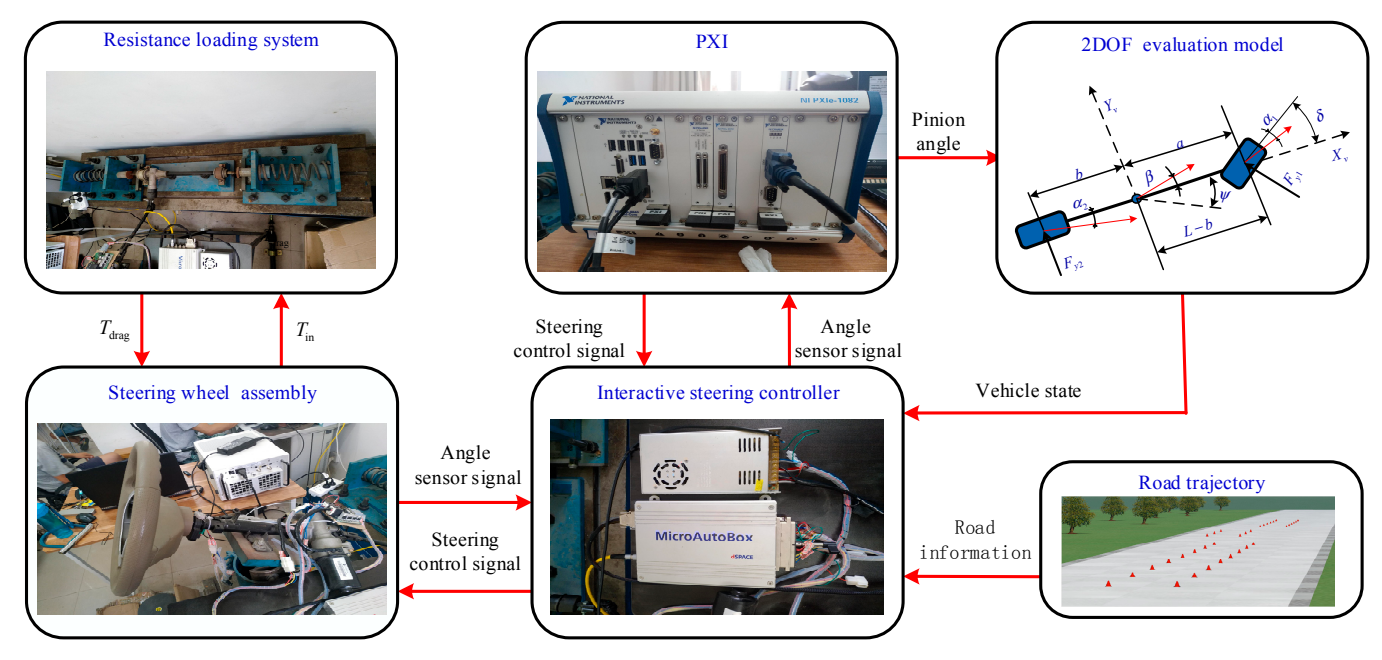

Figure 5. Active steering hardware in the loop test bench.

Figure 6 shows the data collected by the experiment platform and its relevant curves. Figure $6 a$ is the lateral displacement collected under the experiment from which we can see that the vehicle without control produced large lateral displacement when it passed through the double-lane-change route, the vehicle deviated badly from the ideal path, and the vehicle was in a state of instability. The vehicles provided with receding horizon control and PID control both completed the work condition of double-lane-change route; however, the vehicle provided with receding horizon control had a tracking that was closer to the ideal tracking. Figure $6 \mathrm{~b}$ shows the steering wheel angles collected in the process of the experiment. We can see there was a sharp increase in the steering wheel angle of the vehicle without control and that the vehicle lost stability; the vehicles that were provided with receding horizon control and PID control both completed the condition of work. However, the vehicle with receding horizon control had a better response effect and tracking that was closer to the ideal tracking than the vehicle provided with PID control. The vehicle provided with receding horizon control had smaller steering wheel manipulation, which guaranteed better stability of the vehicle. 


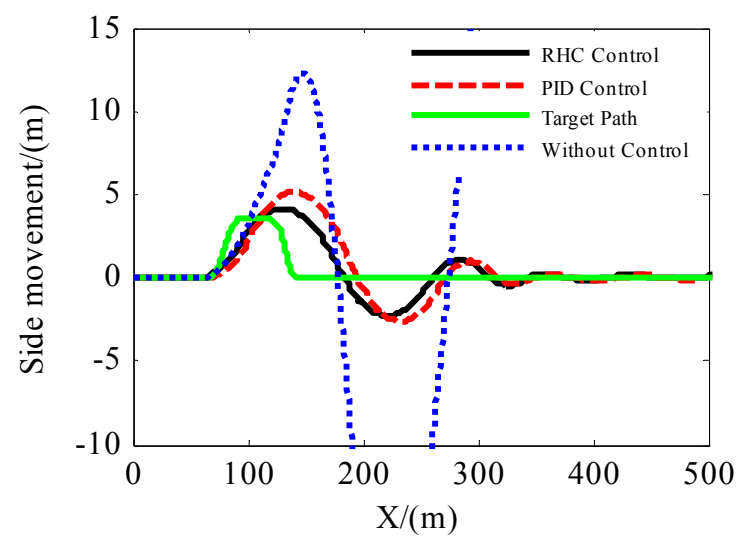

(a)

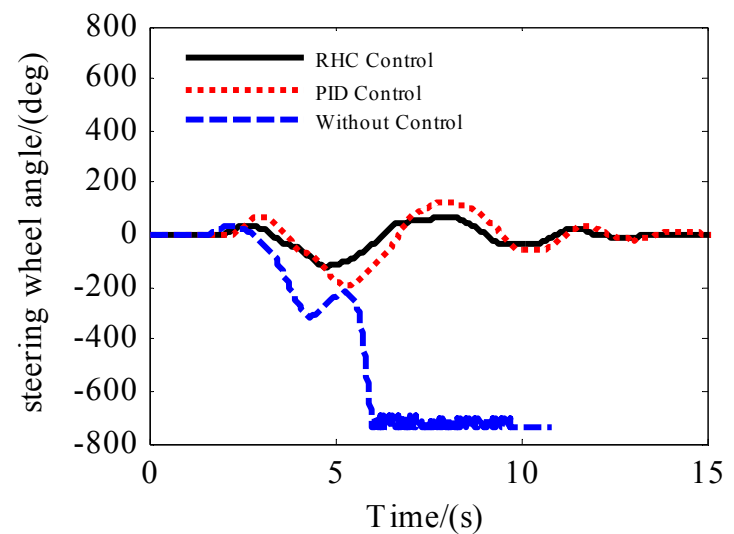

(b)

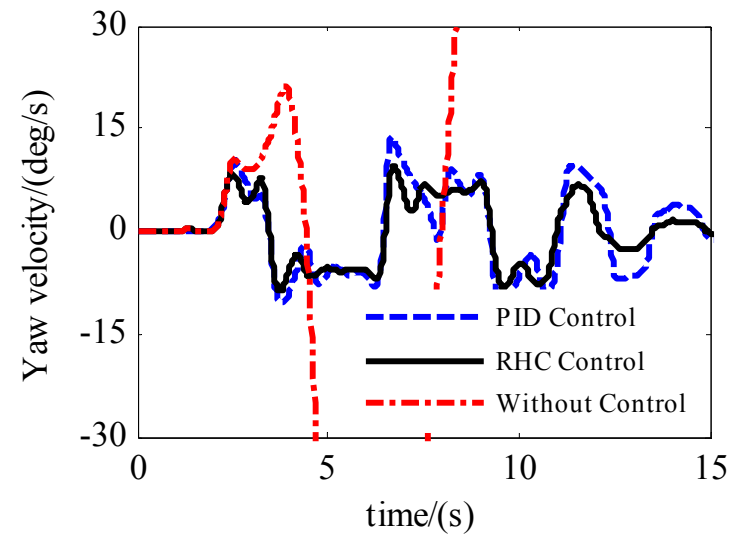

(c)

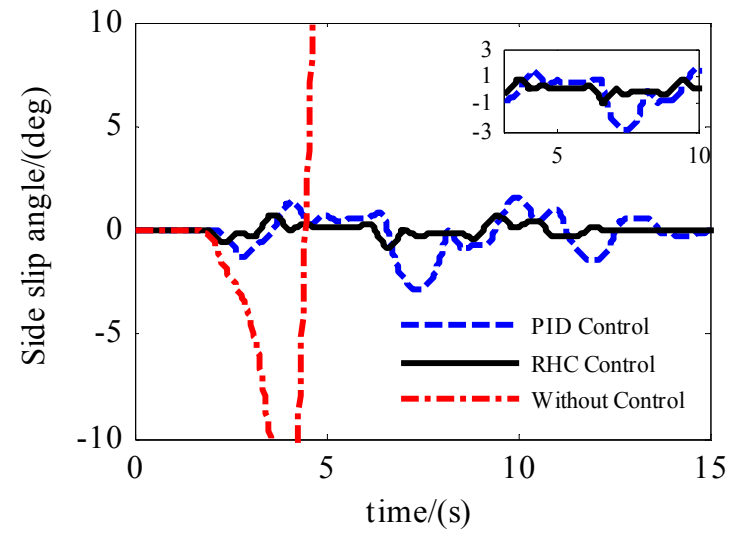

(d)

Figure 6. (a) Lateral displacement; (b) Steering wheel corner; (c) Yaw rate; (d) Sideslip angle. 
Figure $6 \mathrm{c}$ shows the comparison of the yaw rate collected in the process of the experiment. From the figure, it can be seen that the yaw rate of the vehicle without control exceeded the limit range; the yaw rate of the vehicle provided with receding horizon control could be controlled within a certain range, which makes it better for tracking the ideal operating performance of the vehicle. Figure $6 \mathrm{~d}$ shows the comparison of the sideslip angles collected in the experiment. The sideslip angle of the vehicle without control exceeded the limit range. However, the sideslip angles of the vehicles provided with PID control and receding horizon control could be kept within a stable range. The vehicle provided with receding horizon control had a smaller sideslip angle, which implemented better control for stabilizing the vehicle. When the vehicle was driving for $13 \mathrm{~s}$, the vehicle that was provided with receding horizon control completed emergency obstacle avoidance while the PID-controlled vehicle did not completely avoid an emergency obstacle at $15 \mathrm{~s}$.

Figure 7 shows the current of the running motors collected under different control conditions in this experiment. The motor current of the vehicle without control always stayed at a higher value, the current reached the rated limit, and the vehicle lost stability. The current with a PID control was stabilized within the range of $\pm 20 \mathrm{~A}$; however, there was noise fluctuation in the motor current. Compared with the PID control, the vehicle provided with the receding horizon control had less fluctuation in noise of the motor current, a more stable current, and a faster response speed. Figure $7 \mathrm{~d}$ shows the root mean square value of motor currents under different control modes. It can be seen from the figure that the root mean square value of motor current controlled in receding horizon control was the minimum. Therefore, the receding horizon control had a better energy control effect.

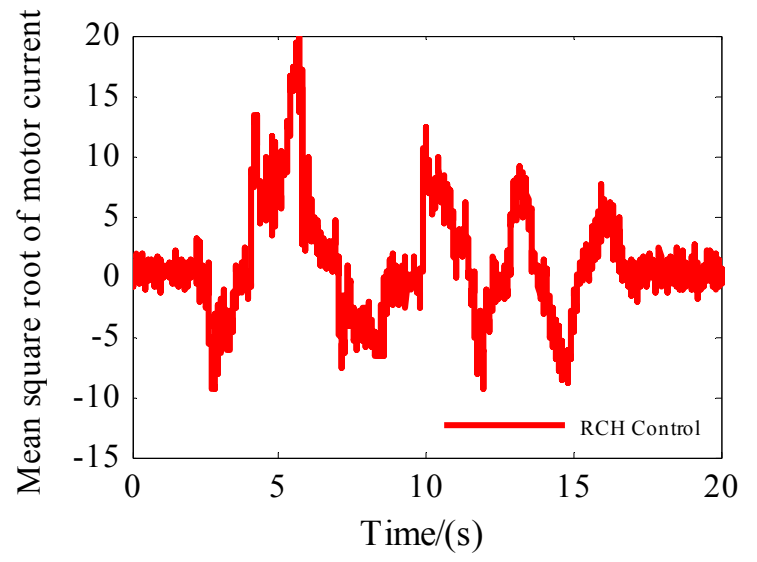

(a)

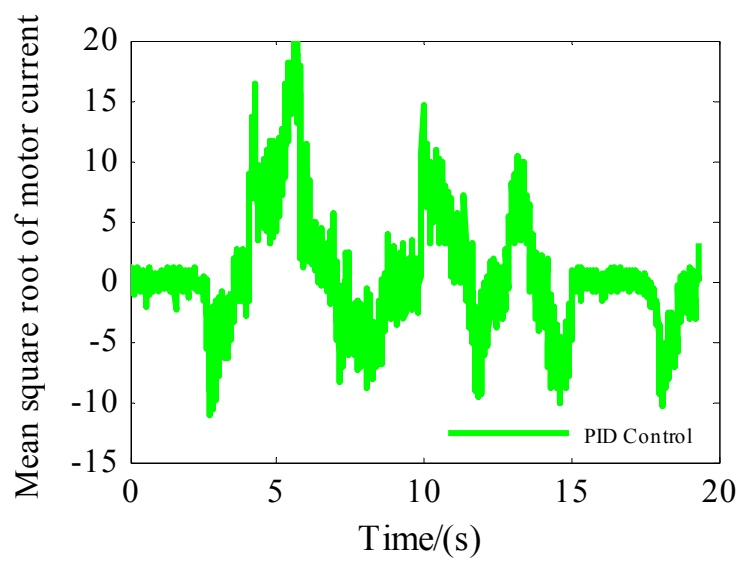

(b)

Figure 7. Cont. 


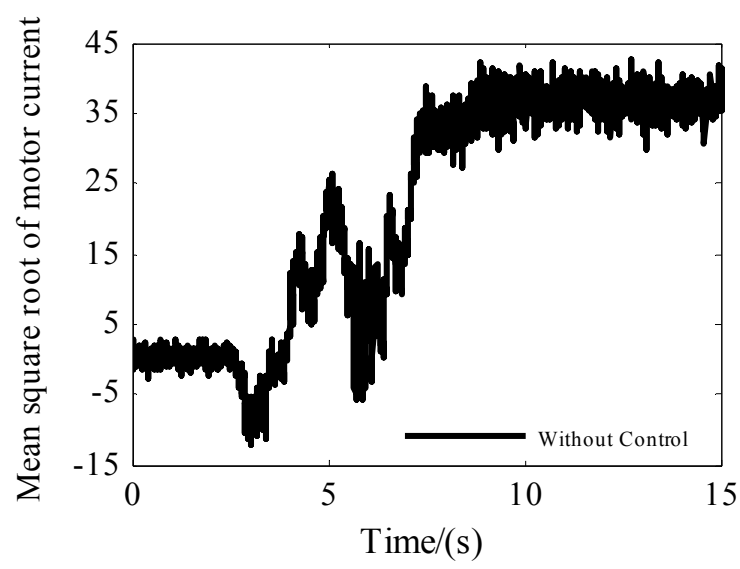

(c)

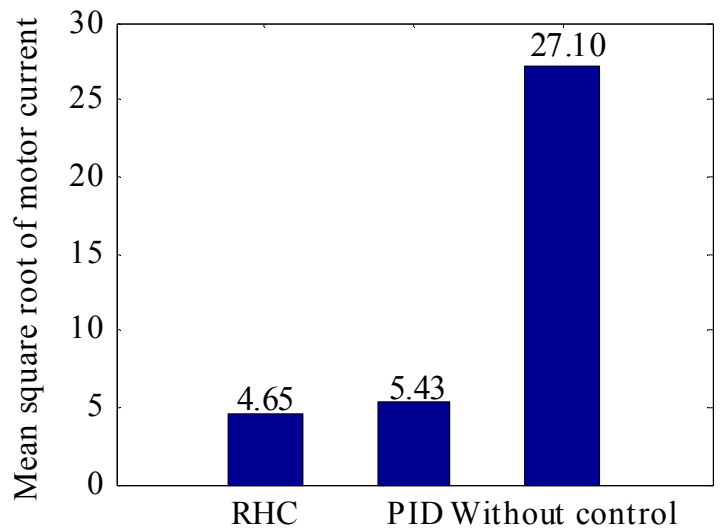

(d)

Figure 7. (a) Receding horizon-controlled current; (b) PID-controlled motor current; (c) Motor current without control; (d) Mean square root value of motor current.

Through the above analysis, the advantages and disadvantages of the two controllers are summarized in Table 2.

Table 2. Advantages and disadvantages of control method.

\begin{tabular}{ccc}
\hline Control Method & Advantages & Disadvantages \\
\hline Receding horizon control & $\begin{array}{c}\text { It can compensate the uncertainty caused } \\
\text { by model mismatch, interference, etc. and } \\
\text { possesses local optimization. }\end{array}$ & $\begin{array}{c}\text { The algorithm is complex and } \\
\text { computationally intensive. }\end{array}$ \\
\hline PID & $\begin{array}{c}\text { It mainly applies to model-free } \\
\text { engineering control and is easy to use. }\end{array}$ & $\begin{array}{c}\text { It has poor robustness and its parameters are } \\
\text { usually regulated by experience; therefore, it } \\
\text { is difficult to obtain optimal PID parameters. }\end{array}$ \\
\hline
\end{tabular}

\section{Conclusions}

In this paper, the receding horizon control was applied to the active steering control, and the design and solving processes of the active steering controller were introduced in detail. The main contributions of this paper can be summarized as follows:

(a) The control model was established based on the nonlinear tire model and the two-freedom model, and the AFS receding horizon controller was designed on the yaw rate deviation.

(b) We built the steering hardware-in-the-loop platform based on LabVIEW RT system to verify the actual control effect of the receding horizon controller. 
The co-simulation results and experimental results of hardware-in-the-loop showed that the designed controller could implement stability control of the vehicle more effectively in the actual system, and improve the performance and stability of the system. In this paper, the main factors affecting the AFS system were considered. The change in system stiffness, the perturbation of the motion, and the parameters of the system were not considered. The next step will be to consider more indicators to establish a more eligible control system.

Author Contributions: J.W. and J.F. conceived and designed the experiments; S.L., J.F. and G.X. performed the experiments; J.F. and C.B. analyzed the data; C.B., S.C. and J.W. contributed reagents/materials/analysis tools; J.F. wrote the paper.

Funding: This research received no external funding.

Acknowledgments: The authors acknowledge support from the Natural Science Foundation of Shandong Province (ZR2016EEQ06) and the National Science and Technology Support Program (2015BAG02B01).

Conflicts of Interest: The authors declare that there are no conflicts of interest regarding the publication of this paper.

\section{References}

1. Jalali, M.; Khosravani, S.; Khajepour, A.; Chen, S.K.; Litkouhi, B. Model predictive control of vehicle stability using coordinated active steering and differential brakes. Mechatronics 2017, 48, 30-41. [CrossRef]

2. Iwano, K.; Raksincharoensak, P.; Nagai, M. A study on shared control between the driver and an active steering control system in emergency obstacle avoidance situations. In Proceedings of the 19th World Congress of the International Federation of Automatic Control, Cape Town, South Africa, 24-29 August 2014.

3. Marino, R.; Scalzi, S.; Netto, M. Integrated driver and active steering control for vision-based lane keeping. Eur. J. Control 2012, 18, 473-484. [CrossRef]

4. Jing, H.; Wang, R.; Chadli, M.; Hu, C.; Yan, F.; Li, C. Fault-Tolerant Control of Four-Wheel Independently Actuated Electric Vehicles with Active Steering Systems. IFAC-PapersOnLine 2015, 48, 1165-1172. [CrossRef]

5. Pérez, J.; Busturia, J.M.; Mei, T.X.; Vinolas, J. Combined active steering and traction for mechatronic bogie vehicles with independently rotating wheels. Annu. Rev. Control 2004, 28, 207-217. [CrossRef]

6. Wu, J.; Cheng, S.; Liu, B.; Liu, C. A Human-Machine-Cooperative-Driving Controller Based on AFS and DYC for Vehicle Dynamic Stability. Energies 2017, 10, 1737. [CrossRef]

7. Wu, J.; Wang, X.; Li, L.; Du, Y. Hierarchical control strategy with battery aging consideration for hybrid electric vehicle regenerative braking control. Energy 2018, 145, 301-312. [CrossRef]

8. Ji, X.; Wu, J.; Zhao, Y.; Liu, Y.; Zhao, X. A new robust control method for active front steering considering the intention of the driver. Proc. Inst. Mech. Eng. Part D J. Auto. Eng. 2015, 229, 518-531. [CrossRef]

9. Wu, J.; Zhao, Y.; Ji, X.; Liu, Y.; Yin, C. A modified structure internal model robust control method for the integration of active front steering and direct yaw moment control. Sci. China Technol. Sci. 2014, 58, 75-85. [CrossRef]

10. Yu, L.; Yun, P.; Bao, C.; Yin, J.; Zheng, Y.; Li, Y. Lateral stability analysis of linear steering system based on fuzzy PID control. J. Shandong Jiaotong Univ. 2014, 22, 4-7.

11. Wang, N.; Shi, J.; Li, G. Research on active steering control strategy of linear control steering system. Pract. Automot. Technol. 2016, 72-73.

12. Li, J.; Yu, C. Vehicle chassis integrated control system based on fuzzy and PID. J. Jilin Univ. 2013, 43, 509-513.

13. Li, Y.; Chen, H.; Gao, B. Application of self-disturbance rejection control in active steering control of front wheels. Automot. Eng. 2011, 33, 388-391.

14. Sang, N.; Wei, M. Active front wheel steering and active suspension control methods. J. Nanjing Univ. Sci. Technol. 2017, 41, 165-172.

15. Huo, C.; Cheng, Y.; Wang, J.; Wu, Y.; Wang, Y. Active steering control study based on genetic algorithm optimization. J. Mech. Electr. Eng. 2016, 33, 122-126.

16. Wu, J.; Zhao, Y.; Ji, X.; Liu, Y.; Zhang, L. Generalized internal model robust control for active front steering intervention. Chin. J. Mech. Eng. 2015, 28, 285-293. [CrossRef]

17. Chen, $\mathrm{W}$; Sun, $\mathrm{Q}$. The first long treasure. $\mathrm{h}_{\infty}$ integrated control of automotive power steering and active suspension system. J. Vib. Eng. 2007, 20, 45-51. 
18. Zhao, W.; Zhang, H.; Li, Y. Displacement and force coupling control design for automotive active front steering system. Mech. Syst Signal Process. 2018, 106, 76-93. [CrossRef]

19. Nam, K.; Oh, S.; Fujimoto, H.; Hori, Y. Robust yaw stability control for electric vehicles based on active front steering control through a steer-by-wire system. Int. J. Automot. Technol. 2012, 13, 1169-1176. [CrossRef]

20. Ji, P.; Shen, B.; Chen, H.; Zhang, T. Research on robust control of active steering system. Automot. Eng. 2013, 35, 1092-1098.

21. Xu, Z. Study on Stability Control Strategy of Vehicle Active Collision Avoidance System. Master's Thesis, Nanjing University of Aeronautics and Astronautics, Nanjing, China, 2017.

22. Mammar, S.; Baghdassarian, V.B. Two-degree-of-freedom formulation of vehicle handling improvement by active steering. In Proceedings of the American Control Conference, Chicago, IL, USA, 28-30 June 2000; pp. 105-109.

23. Chen, H. Model Predictive Control; Science Publishing House: Beijing, China, 2013.

24. Yu, Z. The Theory of Automobiles, 5th ed.; Mechanical industry Press: Beijing, China, 2009.

25. Chen, J.; Li, L.; Song, J. Research on vehicle stability control based on LTV-MPC. Automot. Eng. 2016, 38, 308-316.

26. Wu, J. Study On the Integrated Control Method of Vehicle State and Parameter Estimation and Stability. Ph.D. Thesis, Nanjing University of Aeronautics and Astronautics, Nanjing, China, 2015.

27. Zheng, H.; Zhao, M. Development a hil test bench for electrically controlled steering system. SAE Tech. Pap. Ser. 2016. [CrossRef]

28. Li, X.; Li, Y. Research and development of the simulation platform of the nose wheel with digital steering system based on hardware in the loop. In Proceedings of the 2016 IEEE International Conference on Aircraft Utility Systems (AUS), Beijing, China, 10-12 October 2016.

29. Soltani, A.; Assadian, F. A Hardware-in-the-Loop Facility for Integrated Vehicle Dynamics Control System Design and Validation. IFAC-PapersOnLine 2016, 49, 32-38. [CrossRef]

(C) 2018 by the authors. Licensee MDPI, Basel, Switzerland. This article is an open access article distributed under the terms and conditions of the Creative Commons Attribution (CC BY) license (http:/ / creativecommons.org/licenses/by/4.0/). 